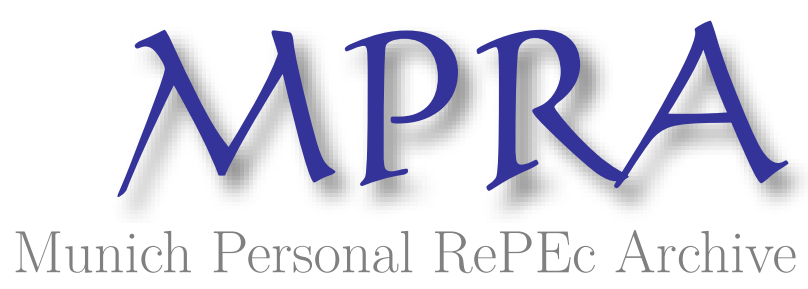

\title{
Expectations, Price Fluctuations and Lorenz Attractor
}

Olkhov, Victor

TVEL, Kashirskoe sh. 49, Moscow, 115409, Russia

20 September 2018

Online at https://mpra.ub.uni-muenchen.de/89105/

MPRA Paper No. 89105, posted 23 Sep 2018 01:30 UTC 


\title{
Expectations, Price Fluctuations and Lorenz Attractor
}

\author{
Victor Olkhov \\ TVEL, Kashirskoe sh. 49, Moscow, 115409, Russia \\ victor.olkhov@gmail.com
}

\begin{abstract}
This paper describes expectations and Buy-Sell transactions of selected Stokes between economic agents and Exchange on economic space as ground for modeling trading volume and price fluctuations. We study simple model of mutual relations between transactions and expectations and derive economic equations that describe disturbances of price, trading volume and expectations. We obtain simple harmonic oscillations for price fluctuations. We show that our model economic equations can take form of Lorenz attractor. Our approximation of transactions and expectations and economic equations on disturbances of price, trading volume and expectations allows apply dynamical systems methods for modeling chaotic behavior of economic and financial systems.
\end{abstract}

Keywords: Financial Transactions; Expectations; Economic Space; Stochastic Prices JEL: C02, C60, E32, F44, G00

This research did not receive any specific grant or financial support from TVEL or funding agencies in the public, commercial, or not-for-profit sectors. 


\section{Introduction}

Price forecasting seems to be the Holy Grail of financial markets and the most valuable problem of macroeconomic and financial theory. Thousands of studies argue different properties of stock price evolution, volatility and returns and any review of current state of research will be non complete and could be enlarged. Thus our introductory sketch presents only small part of references on these problems.

Roughly speaking, there are two ways to describe any complex phenomena. The first way extrapolates behavior of the process under consideration without attempt to understand and describe its internal nature and relations. These methods can use regular hypothesis and develop linear or non-linear extrapolating models to reproduce alikeness, similarity and resemblance of price evolution and fluctuations based on "history" data. Such approaches use stochastic models to describe spectrum or stochastic momentums of price, returns or volatility fluctuations. The second way to describe price behavior is to model the origin and the nature of processes those induce and govern stock price trends and fluctuations as well as trends and fluctuations of returns and volatility. Description and approximation of the processes those impact fluctuations of prices, returns and volatility are much more complex problem than extrapolation of the observed trends and fluctuations. But only the second way has option to describe real economic and financial processes and improve regulations of markets and forecasting of stock price trends and fluctuations.

Most studies on modeling market price belong to the first way. General problems of price fluctuations are studied by (Fama, 1965; Fama, 1970; Lucas, 1978; Kydland and Prescott 1980; Pearce, 1983; Friedman and Laibson, 1989; Campbell and Cochrane, 1995; Heaton and Lucas, 2000; Balke and Wohar, 2001; Hansen, 2013; Cochrane, 2017). Due to Fama (1965) the problem of price forecasting should respond: "To what extent can the past history of a common stock's price be used to make meaningful predictions concerning the future price of the stock? Answers to this question have been provided on the one hand by the various chartist theories and on the other hand by the theory of random walks." Stochastic modeling of price fluctuations (Lucas, 1978) attracted researchers in statistical physics and that resulted in numerous theoretical and experimental studies on statistical properties of price and return fluctuations (Plerou et al., 1999; Shiryaev, 1999; Andersen et al., 2001; Plerou et a., 2001; Gabaix et al., 2003; Alejandro-Quinones et al, 2006; Andersen et al., 2006; Hördahl and Packer, 2007; Kaihatsu and Kurozumi, 2010; Greenwald, Lettau and Ludvigson, S., 2014). Complexity of price fluctuations description induces application of fractal models a studies of 
price and return scaling behavior (Mandelbrot, 1963; Mandelbrot, Fisher, Calvet, 1997; Plerou et al., 1999; Calvet and Fisher, 2002; Gabaix et al., 2003; Carbone, Castelli, Stanley, 2004; Stanley, Plerou and Gabaix, 2008; Segnon and Lux, 2013). Many studies describe properties of return fluctuations (Campbell, 1985; Keim and Stambaugh, 1986; Fama, E.F., 1998; Calvet and Fisher, 2002; Greenwood and Shleifer, 2014; van Binsbergen, Gontis et al, 2016; Gontis et al, 2016; Koijen, 2017) and volatility effects (Bates, 1996; Andersen and Lund, 1997; Andersen et al., 2001; Poon and Granger, 2003; Andersen et al., 2006; Bayraktar, Horst and Sircar, 2008). One of most important contributions to studies of price evolution concern introduction and description of expectations and behavioral models and studies of their impact on price and return fluctuations (Muth, 1961; Fama, 1965; Lucas, R.E., 1972; Sargent and Wallace, 1976; Blume, Easley, 1984; Grandmont,1992; Caporin, Corazzini and Costola, 2014; Greenwood and Shleifer, 2014; Greenwood and Shleifer, 2014; Lof, M., 2014; Thaler, 2018). Let's outline studies that proposed deterministic models as solution for the random price origin. Works by (Barnett and Chen, 1988; Brock and Sayers, 1988; Brock and Hommes, 1997; 1998) proposed that stochastic behavior of price fluctuations have origin in chaotic properties of deterministic dynamical systems. In other words, nonlinearity of deterministic dynamical systems cause observed chaotic dynamic evolution (Lorenz, 1963; Takens, 1981; Barnett and Chen, 1988; Nicolis and Prigogin, 1989; Guckenheimer and Holmes, 1990; Bullard and Butler, 1991; Grandmont, J.M., 1992; Kemp, 1997; Schuster and Just, 2005; Goldstein, J., 2011).

We propose that the price evolutions and fluctuations are the result of collective actions of numerous factors described in the references mentioned above. It is impossible to develop theory that takes into account all possible factors starting with "the past history of a stock's price", action of different shocks like market, technology, economic, political, different models of random walks and disturbances. Any model can describe only part of real factors and observations.

Our paper suggests economic model that takes into account only few factors that impact price fluctuations. General Occam's razor principle (Baker, 2007) states: "Entities are not to be multiplied beyond necessity" and we don't use any general equilibrium assumptions and models on state of markets, prices and etc. To obtain our results we derive simple deterministic economic equations that describe evolution and fluctuations of trading volume and value of transactions for particular Stocks at Exchange. We propose that trade decisions and transactions are made under definite expectations and our economic equations describe impact of these expectations on volume and trading value. For simple approximations we 
derive solutions for volume and trading value and obtain relations for price evolution and fluctuations. We assume that interdependence between expectations and transactions can cause nonlinear coupling of simple economic equations and that results in chaotic price evolution and fluctuations.

We model market transactions on economic space (Olkhov, 2016a; 2016b; 2017a-c). We regard all participants of economic relations and market trades - huge banks, corporations and investment funds, households and small companies and etc., - as economic agents. We assume that agents perform their transactions under action of some risks. Let's propose that it is possible to make risk assessments for all economic agents and such risk assessments provide risk ratings for all agents. Let's use agent's risk ratings as their coordinates on economic space. To simplify the problem let's assume that all agents can perform Buy-Sell transactions of selected Stocks at certain Exchange. Usage of agent's risk ratings as coordinates allows distribute agents and their transactions over economic space. We propose that each transaction defines volume and value of the deal with selected Stocks and each transaction depends upon particular sort of expectations. We show that usage of economic space permits derive economic equations that describe mutual dependence between transactions and expectations on economic space. For simple model we derive solutions of economic equations and obtain representations for volume and value of selected Stocks and obtain prices and their fluctuations. We argue how dependence of expectations on transactions or other expectations can cause nonlinear coupling of simple economic equations and could results in chaotic behavior of price and return fluctuations.

The rest of the paper unfolds as follows. In Section 2 we argue definition of economic space (Olkhov 2016a; 2016b; 2017b; 2017c) and explain how economic space helps for description of transactions and expectations of economic agents. Each Buy-Sell transaction performed by agents at Exchange determines volume and value of selected Stocks traded by this transaction. Thus transactions determine price for each particular trade. We derive system of economic equations that describes evolution of transactions on economic space and hence describe evolution of transactions prices. In Sec. 3 we argue expectations of agents that impact transactions and prices and explain how expectations can be described as functions of time and coordinates on economic space. In Sec.4 we use economic equations to define a simple model of mutual dependence between transactions and expectations and derive simple relations for price fluctuations. In Sec.5 we derive equations (8.1-8.4) on disturbances of trading volume $q(t)$, price $\pi(t)$ and disturbances of expectations $e x_{q}(t)$ and $e x_{s v}(t)$. Further we show that equations $(8.1-8.3)$ on disturbances of trading volume $q(t)$, price $\pi(t)$ and 
expectations $e x_{q}(t)$ can take form of Lorenz attractor (10.1-10.3). Conclusions are in Section 6. Appendix A presents derivations of model economic equation and simple harmonic oscillations of price fluctuations.

\section{Model Setup}

In this section we briefly introduce economic space (Olkhov, 2016a-b; 2017a-d) as ground for modeling price fluctuations. We underline that modeling on economic space have nothing common with spatial economics (Hotelling, 1929; Henderson et al, 2016).

\subsection{Economic space}

Let's regard any traders at certain Exchange like banks, funds, companies, households and etc., as economic agents. We treat agents as simple units of economic and financial system that can execute Buy-Sell transactions. Each agent can execute transaction with particular Stocks at Exchange. Each Buy-Sell transaction determines volume of Stocks purchased and value of this transaction and hence defines the price of Stocks for particular transaction. It is difficult to describe evolutions of transactions for each particular agent. To simplify the problem we propose distribute all agents over points of economic space that we define below. Aggregation of transactions performed by agents at point $\boldsymbol{x}$ of economic space allows transfer the description of Buy-Sell transactions executed by separate agents to description of transactions performed by all agents at point $\boldsymbol{x}$ of economic space. Such approximation allows regard transactions as "continuous transactions media" on economic space. That helps derive economic equations that model evolution of transactions and hence model dynamics and fluctuations of prices. We propose use risks that impact agents and their transactions and assessment of agent's risks as ground for economic space definition.

There are a lot of risks that impact economic agents like credit risks, inflation risks, market risks and many other (Wilier, 1901; Horcher, 2005; McNeil, Frey and Embrechts, 2005; Skoglund and Chen, 2015). We don't argue specific economic or financial risks but treat them all as factors that impact economic agents, their transactions and entire economics. All agents are at risk but not for all risks and not for all agents risk assessments are provided. For large banks and corporations risk assessments are provided by rating companies as Moody's, Fitch, S\&P (Metz and Cantor, 2007; Chane-Kon, et.al, 2010; Kraemer and Vazza, 2012). Ratings take value of risk grades as $A A A, A, B B, C$ and etc., and follow the risk rating methodologies (Altman, 2010; Staff U.S.SEC, 2012; S\&P, 2014; Pitman and Moss, 2016). We propose (Olkhov, 2016a; 2016b; 2017a-d) regard risk grades $A A A, A, B B, C$ and etc., as 
discrete points $x_{1}, \ldots x_{m}$ of economic space. Such economic space imbed economic agents by their risk ratings $\boldsymbol{x}$. Ratings of single risk distribute agents over points of one-dimensional economic space. Assessments of two or more risks distribute agents by their risk ratings on economic space with dimension two or more. Let's assume that risk assessment methodology can utilize continuous risk grades. One can always assume that risk grades take value between most secure grade equals $O$ and most risky grade equals $X$. Assessments of agent's ratings for single risk fill interval $(O, X)$ of economic domain on space $R$. Assessments of agent's ratings for $n$ risks define agent's coordinates on economic domain on $R^{n}$. Let's propose that econometrics provide sufficient data for risk assessments of all economic agents. This assumption doesn't impose restrictions or ad hoc requirements on economic system under consideration like assumptions made by general equilibrium hypothesis (Arrow, 1974; Starr, 2011). We just assume that development of econometrics and risk methodologies similar to quality and granularity of U.S. National Income and Product Accounts system (Fox, et al., 2014) can solve the problem of risk assessment for most economic agents. Let's propose that macroeconomics is under action of $n=1,2,3$.. risks and risk assessments of economic agents distribute them over economic domain on economic space $R^{n}$. For additional details on economic space modeling we refer to (Olkhov, 2016a - 2018). For brevity let's further note economic space as e-space and economic agents as e-particles (economic particles). We use roman letters $f$, $t$, etc., to define scalar and bold letters $\boldsymbol{x}, \boldsymbol{B}, \boldsymbol{P}$, etc., to define vector variables and functions.

\subsection{Transactions on e-space}

To describe properties of price evolution and fluctuations let's consider particular Stocks that are traded at Exchange. Each transaction $\boldsymbol{t r}_{i}(t, \boldsymbol{x})$ executed by agent $i$ at point $\boldsymbol{x}$ at moment $t$ with selected Stocks determine trading volume $Q_{i}(t, \boldsymbol{x})$ and Stock value $S V_{i}(t, \boldsymbol{x})$ of transaction. Let's define transactions $\operatorname{tr}_{i}(t, \boldsymbol{x})$ as two component functions and use bold letters:

$$
\boldsymbol{t r}_{i}(t, \boldsymbol{x})=\left(Q_{i}(t, \boldsymbol{x}) ; S V_{i}(t, \boldsymbol{x})\right)
$$

Price $p_{i}(t, \boldsymbol{x})$ of this transaction is determined by obvious relation (1.2):

$$
S V_{i}(t, \boldsymbol{x})=p_{i}(t, \boldsymbol{x}) Q_{i}(t, \boldsymbol{x})
$$

There are several kinds of transactions between e-particles and Exchange. First, there are transactions those form Exchange Bid-Ask order book. E-particles execute transactions those determine Exchange Bid-Ask order book and transactions those delete previous proposals from Bid-Ask order book. Bid-Ask order book is a ground for Buy-Sell transactions executed 
by e-particles (agents) at Exchange. Below we model transactions of e-particles with Exchange based on available Bid-Ask order book data.

Description of transactions of numerous agents with Exchange is a very complex problem. To simplify this problem let's replace precise description of transactions performed by separate agents by rougher description of transactions executed during time term $\Delta$ by numerous e-particles with coordinates in a unit volume $d V(\boldsymbol{x})$ of e-space. Let's outline that risk coordinates $\boldsymbol{x}=\left(x_{1}, \ldots x_{n}\right)$ of e-particles on economic domain of $n$-dimensional e-space $R^{n}$ are reduced by

$$
0 \leq x_{i} \leq X_{i}, i=1, \ldots n
$$

Here $x_{i}=0$ define most secure and $X_{i}$ define most risky grades for risk $i$. Relations (1.3) define economic domain (Olkhov, 2017d; 2018) and all economic agents have their "risk" coordinates at economic domain (1.3). Let's assume that a unit volume $d V(\boldsymbol{x})$ at point $\boldsymbol{x}$ contains many separate agents but scales $d V_{i}$ of a unit volume are small to compare with scales $X_{i}$ of economic domain.

$$
d V_{i} \ll X_{i}, i=1, \ldots n ; \quad d V=\prod_{i=1, . . n} d V_{i}
$$

Let's aggregate all transactions $\boldsymbol{t r}_{i}(t, \boldsymbol{x})$ of e-particles from a unit volume $d V(\boldsymbol{x})$ near point $\boldsymbol{x}$ during time term $\Delta$ and call it as transaction $\operatorname{Tr}(t, \boldsymbol{x})$ from point $\boldsymbol{x}$. Such approximation neglects excess granularity of separate e-particles on e-space and moves description of transactions $\boldsymbol{t r}_{i}(t, \boldsymbol{x})$ of separate e-particles (agents) to description of transactions $\boldsymbol{T r}(t, \boldsymbol{x})$ as function of points $\boldsymbol{x}$ of e-space. Let's repeat that transactions $\operatorname{Tr}(t, \boldsymbol{x})$ are performed by numerous e-particles in a unit volume $d V(\boldsymbol{x})$ during time term $\Delta$. Time term $\Delta$ can equal one second, hour, day, week and etc., and is determined by particular problem under consideration. This approximation is intermediate between description of transactions of separate agents and description of transactions performed by all e-particles of entire economics. Let's call such intermediate approximation as economic hydrodynamic-like approximation because it is alike to hydrodynamic approximation in physics. Hydrodynamic scales are small to compare with macro scales of fluid flows but each unit volume $d V(\boldsymbol{x})$ contain a lot of physical particles (Landau and Lifshitz, 1987). To define above consideration in a more formal manner let's assume that e-particles on e-space $R^{n}$ at moment $t$ have coordinates $\boldsymbol{x}=\left(x_{1}, \ldots x_{n}\right)$ and velocities $\boldsymbol{v}=\left(v_{1}, \ldots v_{n}\right)$. E-space $R^{n}$ describes evolution of eparticles under action of $n$ risks. Velocities $\boldsymbol{v}=\left(v_{1}, \ldots v_{n}\right)$ describe change of e-particles risk coordinates on e-space $R^{n}$ during time $\Delta$. Transactions $\boldsymbol{t r}_{i}(t, \boldsymbol{x})$ of e-particle $i$ at point $\boldsymbol{x}$ at Exchange are defined by (1.1). Let's define transaction $\boldsymbol{T} \boldsymbol{r}(t, \boldsymbol{x})$ from point $\boldsymbol{x}$ as sum of all 
transactions $\operatorname{tr}_{i}(t, \boldsymbol{x})$ of all e-particles $i$ during time term $\Delta$ in a unit volume $d V(\boldsymbol{x})$ :

$$
\begin{gathered}
\boldsymbol{T r}(t, \boldsymbol{x})=(Q(t, \boldsymbol{x}) ; S V(t, \boldsymbol{x}))=\sum_{i \in d V(\boldsymbol{x}) ; \Delta} \boldsymbol{t r}_{i}(t, \boldsymbol{x}) \\
Q(t, \boldsymbol{x})=\sum_{i \in d V(\boldsymbol{x}) ; \Delta} Q_{i}(t, \boldsymbol{x}) \quad ; \quad S V(t, \boldsymbol{x})=\sum_{i \in d V(\boldsymbol{x}) ; \Delta} S V_{i}(t, \boldsymbol{x}) \\
\operatorname{tr}_{\boldsymbol{i}}(t, \boldsymbol{x})=\left(Q_{i}(t, \boldsymbol{x}) ; S V_{i}(t, \boldsymbol{x})\right)
\end{gathered}
$$

Price $p_{i}(t, \boldsymbol{x})$ of transaction $\boldsymbol{t r}_{i}(t, \boldsymbol{x})$ executed by e-particle $i$ at point $\boldsymbol{x}$ is determined by (1.2). Transaction $\boldsymbol{T} \boldsymbol{r}(t, \boldsymbol{x})$ as function of point $\boldsymbol{x}$ defines price $p(t, \boldsymbol{x})(2.2)$ at point $\boldsymbol{x}$ as

$$
S V(t, \boldsymbol{x})=p(t, \boldsymbol{x}) Q(t, \boldsymbol{x})
$$

Price $p(t, \boldsymbol{x})$ is averaged over prices of transactions performed by agents in a unit volume $d V(\boldsymbol{x})$ at moment $t$ during time $\Delta$. Aggregation by scales of unit volume $d V$ move description of transactions of separate e-particles to description of transactions as function of $\boldsymbol{x}$ on espace. Let's outline that transactions $\boldsymbol{T r}(t, \boldsymbol{x})$ are associated with points $\boldsymbol{x}$ of e-space, but not with separate e-particles. Relations $(2.1 ; 2.2)$ describe transactions $\boldsymbol{T} \boldsymbol{r}(t, \boldsymbol{x})$ as functions of $\boldsymbol{x}$ or as continuous transactions media on e-space. To derive economic equations that describe evolution of transactions $\boldsymbol{T} \boldsymbol{r}(t, \boldsymbol{x})$ one should take into account possible motion of transactions media induced by velocities of separate e-particles on e-space. Indeed, velocities $\boldsymbol{v}_{\boldsymbol{i}}=\left(v_{1}, \ldots v_{n}\right)$ describe change of "risk" coordinates of e-particle $i$ during time $\Delta$. Thus transactions $\boldsymbol{T r}(t, \boldsymbol{x})$ determined by (2.1) can flow on e-space alike to flow of fluids. To describe changes of transactions $\operatorname{Tr}(t, \boldsymbol{x})$ induced by motion of e-particles on e-space let's define transactions "impulses" $\boldsymbol{p}_{\boldsymbol{i}}$ of e-particle $i$ as:

$$
\boldsymbol{p}_{i}(t, \boldsymbol{x})=\left(\boldsymbol{p}_{i Q}(t, \boldsymbol{x}) ; \boldsymbol{p}_{i S V}(t, \boldsymbol{x})\right)=\left(Q_{i}(t, \boldsymbol{x}) \boldsymbol{v}_{\boldsymbol{i}}(t, \boldsymbol{x}) ; S V_{i}(t, \boldsymbol{x}) \boldsymbol{v}_{\boldsymbol{i}}(t, \boldsymbol{x})\right)
$$

Transactions "impulses" $\boldsymbol{p}_{i}(t, \boldsymbol{x})$ are additive and sum of "impulses" of two e-particles 1 and 2 equals sum of group of two e-particles (take into account (2.1)):

$$
\begin{aligned}
& \boldsymbol{p}(t, \boldsymbol{x})=(Q(t, \boldsymbol{x}) \boldsymbol{v}(t, \boldsymbol{x}) ; S V(t, \boldsymbol{x}) \boldsymbol{v}(t, \boldsymbol{x}))=\boldsymbol{p}_{\mathbf{1}}(t, \boldsymbol{x})+\boldsymbol{p}_{\mathbf{2}}(t, \boldsymbol{x}) \\
& Q(t, \boldsymbol{x}) \boldsymbol{v}_{Q}(t, \boldsymbol{x})=Q_{1}(t, \boldsymbol{x}) \boldsymbol{v}_{\mathbf{1}}(t, \boldsymbol{x})+Q_{2}(t, \boldsymbol{x}) \boldsymbol{v}_{\mathbf{2}}(t, \boldsymbol{x}) \\
& S V(t, \boldsymbol{x}) \boldsymbol{v}_{S V}(t, \boldsymbol{x})=S V_{1}(t, \boldsymbol{x}) \boldsymbol{v}_{\mathbf{1}}(t, \boldsymbol{x})+S V_{2}(t, \boldsymbol{x}) \boldsymbol{v}_{\mathbf{2}}(t, \boldsymbol{x}) \\
& \quad Q(t, \boldsymbol{x})=\sum_{i \in d V(\boldsymbol{x}) ; \Delta} Q_{i}(t, \boldsymbol{x}) \quad ; \quad S V(t, \boldsymbol{x})=\sum_{i \in d V(\boldsymbol{x}) ; \Delta} S V_{i}(t, \boldsymbol{x})
\end{aligned}
$$

Relations (2.4.1-2.4.3) show that "risk" velocities $\boldsymbol{v}_{Q}(t, \boldsymbol{x})$ of the Volume $Q(t, \boldsymbol{x})$ of Stocks and velocities $\boldsymbol{v}_{S V}(t, \boldsymbol{x})$ of the Stock Value $S V(t, \boldsymbol{x})$ can be different. Similar to (2.1) we aggregate transactions "impulses" of e-particles in a unit volume $d V(\boldsymbol{x})$ at moment $t$ during time $\Delta$ and define transactions "impulses" and velocities as functions of $\boldsymbol{x}$ :

$$
\boldsymbol{P}(t, \boldsymbol{x})=\left(\boldsymbol{P}_{Q}(t, \boldsymbol{x}) ; \boldsymbol{P}_{S V}(t, \boldsymbol{x})\right)=\sum_{i \in d V(x) ; \Delta} \boldsymbol{p}_{i}(t, \boldsymbol{x})
$$




$$
\begin{aligned}
& \boldsymbol{P}_{Q}(t, \boldsymbol{x})=Q(t, \boldsymbol{x}) \boldsymbol{v}_{Q}(t, \boldsymbol{x})=\sum_{i \in d V(\boldsymbol{x}) ; \Delta} Q_{i}(t, \boldsymbol{x}) \boldsymbol{v}_{i}(t, \boldsymbol{x}) \\
& \boldsymbol{P}_{S V}(t, \boldsymbol{x})=S V(t, \boldsymbol{x}) \boldsymbol{v}_{S V}(t, \boldsymbol{x})=\sum_{i \in d V(\boldsymbol{x}) ; \Delta} S V_{i}(t, \boldsymbol{x}) \boldsymbol{v}_{i}(t, \boldsymbol{x}) \\
& \boldsymbol{p}_{i}(t, \boldsymbol{x})=\left(\boldsymbol{p}_{i Q}(t, \boldsymbol{x}) ; \boldsymbol{p}_{i S V}(t, \boldsymbol{x})\right)=\left(Q_{i}(t, \boldsymbol{x}) \boldsymbol{v}_{i}(t, \boldsymbol{x}) ; S V_{i}(t, \boldsymbol{x}) \boldsymbol{v}_{i}(t, \boldsymbol{x})\right) \\
& \boldsymbol{v}(t, \boldsymbol{x})=\left(\boldsymbol{v}_{Q}(t, \boldsymbol{x}) ; \boldsymbol{v}_{S V}(t, \boldsymbol{x})\right)
\end{aligned}
$$

Economic meaning of "impulses" is very simple. "Impulses" $\boldsymbol{p}_{\boldsymbol{i}}(t, \boldsymbol{x})$ describe flows of transactions of separate agents due to motion of agents on e-space. "Impulses" $\boldsymbol{P}(t, \boldsymbol{x})$ describe flows of transactions media induced by collective "risk" motion of all e-particles in a unit volume $d V(\boldsymbol{x})$ during time $\Delta$. Relations (2.1-2.5.5) define transactions $\boldsymbol{T r}(t, \boldsymbol{x})$, their "impulses" $\boldsymbol{P}(t, \boldsymbol{x})$ and velocities $\boldsymbol{v}(t, \boldsymbol{x})$ as functions of coordinates $\boldsymbol{x}$ on e-space. These relations replace modeling transactions $\boldsymbol{t r}_{i}(t, \boldsymbol{x})$ of separate e-particle $i$ at point $\boldsymbol{x}$ on e-space by description of transactions $\boldsymbol{T} \boldsymbol{r}(t, \boldsymbol{x})$ with less accuracy on e-space determined by coarsening over unit volumes $d V$ during time term $\Delta$. Such treatment has certain parallels to hydrodynamic approximation in physics (Landau and Lifshitz, 1987; Resibois and De Leener, 1977). Hydrodynamic approximation neglect granularity of separate particles and describes physical properties of the system as continuous media or physics of fluids. We develop similar approximation to describe transactions of e-particles (economic agents) on espace. Integral of transactions $\boldsymbol{T r}(t, \boldsymbol{x})$ by variable $\boldsymbol{x}$ over e-space $R^{n}$ defines all transactions $\boldsymbol{T r}(t)$ performed in the entire economics with Exchange at moment $t$.

$$
\begin{aligned}
& \boldsymbol{T r}(t)=(Q(t) ; S V(t))=\int d \boldsymbol{x} \boldsymbol{T} \boldsymbol{r}(t, \boldsymbol{x}) \\
& Q(t)=\int d \boldsymbol{x} Q(t, \boldsymbol{x}) ; S V(t)=\int d \boldsymbol{x} S V(t, \boldsymbol{x})
\end{aligned}
$$

Thus the price $p(t)$ (2.7) of transactions $\boldsymbol{T} \boldsymbol{r}(t)$ performed by all e-particles at moment $t$ equals:

$$
S V(t)=p(t) Q(t)
$$

To describe evolution and fluctuations of the price $p(t)(2.7)$ one should model evolution and fluctuations of (2.1-2.6). Roughening of transactions modeling permits derive economic equations that describe transactions $\boldsymbol{T r}(t, \boldsymbol{x})$, their "impulses" $\boldsymbol{P}(t, \boldsymbol{x})$ and velocities $\boldsymbol{v}(t, \boldsymbol{x})$.

\subsection{Economic equations for transactions}

Definitions of macro variables and transactions as functions of coordinates on e-space and derivation of the economic equations that describe their evolution were presented in (Olkhov, 2016a; 2017a; 2017b; 2017c; 2018). Below we argue derivation of the economic equations for transactions $\boldsymbol{T} \boldsymbol{r}(t, \boldsymbol{x})$. Let's regard transactions $\boldsymbol{T} \boldsymbol{r}(t, \boldsymbol{x})$ of selected Stocks as example only. The same considerations can be applied to description of transactions with portfolios, any Assets and Commodities, transactions that determine Bid-Ask order book or cancel orders 
from Bid-Ask order book and etc.

Let's regard change of transactions $\boldsymbol{T r}(t, \boldsymbol{x})(2.1)$ in a unit volume $d V(\boldsymbol{x})$ at point $\boldsymbol{x}$. $\boldsymbol{T r}(t, \boldsymbol{x})$ can change due to two factors. First factor describes change of $\boldsymbol{T} \boldsymbol{r}(t, \boldsymbol{x})$ in time as $\partial \boldsymbol{T} \boldsymbol{r} / \partial t$. Second factor describes change of $\boldsymbol{T r}(t, \boldsymbol{x})$ in a unit volume $d V(\boldsymbol{x})$ due to flux of transactions flow $\boldsymbol{v} \boldsymbol{T r}$ through surface of a unit volume. Divergence theorem (Strauss 2008, p.179) states that surface integral of flux $\boldsymbol{v} \boldsymbol{T r}$ through surface of a unit volume $d V(\boldsymbol{x})$ equals volume integral of divergence $\boldsymbol{v} \boldsymbol{T r}$. Thus total change of transactions $\boldsymbol{T r}(t, \boldsymbol{x})$ in a unit volume $d V(\boldsymbol{x})$ equals

$$
\frac{\partial}{\partial t} \boldsymbol{T r}(t, x)+\nabla \cdot(\boldsymbol{v}(t, \boldsymbol{x}) \boldsymbol{T r}(t, x))
$$

Here $\boldsymbol{v}$ - velocity of transaction $\boldsymbol{T} \boldsymbol{r}(t, \boldsymbol{x})$ on $n$-dimension e-space $R^{n}$ determined by $(2.1-2.5)$. Taking into account relations (2.5.1-2.5.5) one can present components of $\boldsymbol{T} \boldsymbol{r}(t, \boldsymbol{x})(3.1)$ as

$$
\begin{aligned}
& \frac{\partial}{\partial t} Q(t, \boldsymbol{x})+\nabla \cdot\left(\boldsymbol{v}_{Q}(t, \boldsymbol{x}) Q(t, \boldsymbol{x})\right) \\
& \frac{\partial}{\partial t} S V(t, \boldsymbol{x})+\nabla \cdot\left(\boldsymbol{v}_{S V}(t, \boldsymbol{x}) S V(t, \boldsymbol{x})\right)
\end{aligned}
$$

Divergence $\nabla \cdot$ equals:

$$
\nabla \cdot\left(\boldsymbol{v}_{Q}(t, \boldsymbol{x}) Q(t, \boldsymbol{x})\right)=\sum_{i=1, \ldots n} \frac{\partial}{\partial x_{i}}\left(v_{Q i}(t, \boldsymbol{x}) Q(t, \boldsymbol{x})\right)
$$

Change of transactions $\operatorname{Tr}(t, \boldsymbol{x})$ can be induced by action of different factors. Let's note them as $\boldsymbol{F}_{1}$. Then equation on transactions $\boldsymbol{T} \boldsymbol{r}(t, \boldsymbol{x})$ takes form:

$$
\frac{\partial \boldsymbol{T r}}{\partial t}+\nabla \cdot(\boldsymbol{v} \boldsymbol{T r})=\boldsymbol{F}_{1} ; \boldsymbol{F}_{1}=\left(F_{1 Q} ; F_{1 S V}\right)
$$

or for components of transaction: for Volume $Q(t, \boldsymbol{x})$ and for Stock Value $S V(t, \boldsymbol{x})$ economic equations take form:

$$
\frac{\partial Q}{\partial t}+\nabla \cdot\left(\boldsymbol{v}_{Q} Q\right)=F_{1 Q} ; \frac{\partial S V}{\partial t}+\nabla \cdot\left(\boldsymbol{v}_{S V} S V\right)=F_{1 S V}
$$

Equations $(3.4 ; 3.5)$ describe a simple balance between evolution of transaction $\boldsymbol{T r}(t, \boldsymbol{x})$ at point $\boldsymbol{x}$ and other factors that impact change of transactions. Left side (3.4) describes change in time and due to flux through surface of a unit volume. Right side describes impact of other factors. The same reasons define equations on transactions impulses $\boldsymbol{P}(t, \boldsymbol{x})$ determined by $(2.5 .1-2.5 .5)$ as:

$$
\begin{gathered}
\frac{\partial \boldsymbol{P}}{\partial t}+\nabla \cdot(\boldsymbol{v} \boldsymbol{P})=\boldsymbol{F}_{2} ; \quad \boldsymbol{F}_{2}=\left(\boldsymbol{F}_{2 Q} ; \boldsymbol{F}_{2 S V}\right) \\
\frac{\partial \boldsymbol{P}_{Q}}{\partial t}+\nabla \cdot\left(\boldsymbol{v}_{Q} \boldsymbol{P}_{Q}\right)=\boldsymbol{F}_{2 Q} ; \frac{\partial \boldsymbol{P}_{S V}}{\partial t}+\nabla \cdot\left(\boldsymbol{v}_{S V} \boldsymbol{P}_{S V}\right)=\boldsymbol{F}_{2 S V}
\end{gathered}
$$

Left sides of $(3.6 .1 ; 3.6 .2)$ describe change of transactions impulses $\boldsymbol{P}(t, \boldsymbol{z})$ (2.5.1) due to change in time $\partial \boldsymbol{P} / \partial t$ and due to flux $\boldsymbol{v P}$ through surface of unit volume that equal divergence $\nabla \cdot(\boldsymbol{v P})$. Right-hand side $\boldsymbol{F}_{2}$ describes action of other factors that impact on transactions 
impulses $\boldsymbol{P}(t, \boldsymbol{x})$. Economic equations $(3.4 ; 3.5 ; 3.6 .1$; 3.6.2) present formal relations that describe evolution of transactions $\boldsymbol{T r}(t, \boldsymbol{x})$ and their impulses $\boldsymbol{P}(t, \boldsymbol{x})$. To study a reasonable economic model with equations (3.4-3.6.2) one should define right side factors $\boldsymbol{F}_{\boldsymbol{1}}$ and $\boldsymbol{F}_{2}$.

\section{Expectations as functions on e-space}

Let's use equations (3.4-3.6.2) to model evolution and fluctuations of Transactions of selected Stocks at Exchange under action of Expectations. To do that let's argue Expectations of separate e-particles (agents) and their impact on Transactions executed by agents. Let's study a simple model: Transactions are executed under action of Expectations. Expectations are formed by current macroeconomic variables and Transactions. As we argue in (Olkhov, 2017b-c; 2018) Transactions define evolution of macroeconomic variables. Thus it is reasonable to assume that Expectations depend on Transactions only. To model relations between Transactions and Expectations we present transition from description of Expectations of separate agents to description of Expectations as functions of $\boldsymbol{x}$ on economic domain of e-space similar to Sec.2.

\subsection{Expectations as functions on e-space}

Let's call $\boldsymbol{e} \boldsymbol{x}_{i}(t, \boldsymbol{x})$ as Expectations of e-particle $i$ (economic agent) at point $\boldsymbol{x}$. Expectations are treated as factors that impact price fluctuations at least since Muth (1961) and were studied by Lucas (1972) and in numerous publications (Sargent and Wallace, 1976; Hansen and Sargent, 1979; Kydland and Prescott, 1980; Blume and Easley, 1984; Brock and Hommes, 1998; Manski, 2004; Brunnermeier and Parker, 2005; Dominitz and Manski, 2005; Klaauw et al, 2008; Janžek and Ziherl, 2013; Caporin, Corazzini and Costola, 2014; Greenwood and Shleifer, 2014; Lof, 2014; Manski, 2017; Thaler, 2018). There are a lot of studies on measuring expectations (Manski, 2004; Dominitz and Manski, 2005; Klaauw et al, 2008; Bachmann and Elstner, 2013; Janžek and Ziherl, 2013; Manski, 2017; Tanaka et al, 2018). We propose that expectations are measured as probabilities and define execution of transactions $\boldsymbol{t r}_{i}(t, \boldsymbol{x})$ under considerations. We don't specify particular type of expectations $\boldsymbol{e x}_{i}(t, \boldsymbol{x})$ like expected returns, expected inflation and etc. As first approximation let's propose that all e-particles (agents) that execute transactions $\operatorname{tr}_{i}(t, \boldsymbol{x})$ at Exchange have same, the only sort of expectations $\boldsymbol{e x}_{i}(t, \boldsymbol{x})$ that approve performance of transactions $\boldsymbol{t r}_{i}(t, \boldsymbol{x})$. Actually we model impact of expectations $\boldsymbol{e x}_{i}(t, \boldsymbol{x})$ on transactions $\boldsymbol{t r}_{i}(t, \boldsymbol{x})$ only. 
Let's state that the only sort of expectations $\boldsymbol{e x}_{i}(t, \boldsymbol{x})$ of e-particles $i$ at point $\boldsymbol{x}$ have "weights" proportional to their transactions $\boldsymbol{t r}_{i}(t, \boldsymbol{x})$. We define transactions as (1.1) and hence let's define expectations $\boldsymbol{e x}_{i}(t, \boldsymbol{x})$ of e-particles $i$ as two component function

$$
\boldsymbol{e x}_{i}(t, \boldsymbol{x})=\left(e x_{i Q}(t, \boldsymbol{x}) ; \operatorname{ex}_{i S V}(t, \boldsymbol{x})\right)
$$

Let's assume that expectations $e x_{i Q}(t, \boldsymbol{x})$ are responsible for decision on trading volume $Q_{i}(t, \boldsymbol{x})$ of transaction (1.1) and expectations $\operatorname{ex}_{i S V}(t, \boldsymbol{x})$ are responsible for decision on Stocks value $S V_{i}(t, \boldsymbol{x})$ of transaction (1.1). To aggregate expectations $\boldsymbol{e x}_{i}(t, \boldsymbol{x})$ of all e-particles with coordinates $\boldsymbol{x}$ in a unit $d V(t, \boldsymbol{x})$ during time $\Delta$ let's define "expected transactions" $\boldsymbol{e t}_{i}(t, \boldsymbol{x})$ as expectations $\boldsymbol{e x}_{i}(t, \boldsymbol{x})$ "weighted" by transaction $\boldsymbol{t r}_{i}(t, \boldsymbol{x})$.

$$
\boldsymbol{e t}_{i}(t, \boldsymbol{x})=\boldsymbol{e x}_{i}(t, \boldsymbol{x}) \boldsymbol{t r}_{\boldsymbol{i}}(t, \boldsymbol{x})=\left(e x_{i Q}(t, \boldsymbol{x}) Q_{i}(t, \boldsymbol{x}) ; e x_{i S V}(t, \boldsymbol{x}) S V_{i}(t, \boldsymbol{x})\right)
$$

Relations (4.2) define additive expected transactions $\boldsymbol{e t}_{i}(t, \boldsymbol{x})$. Additivity of (4.2) is required to derive expected transaction of group of e-particles as sum of their expected transactions. That allows define expected transactions $\boldsymbol{E} \boldsymbol{T}(t, \boldsymbol{x})$ of group of e-particles with coordinates in a unit $d V(t, \boldsymbol{x})$ during time $\Delta$ as:

$$
\begin{gathered}
\boldsymbol{E} \boldsymbol{T}(t, \boldsymbol{x})=\boldsymbol{E} \boldsymbol{x}(t, \boldsymbol{x}) \boldsymbol{T r}(t, \boldsymbol{x})=\left(E T_{Q}(t, x) ; E T_{S V}(t, x)\right) \\
E T_{Q}(t, x)=E x_{Q}(t, \boldsymbol{x}) Q(t, \boldsymbol{x})=\sum_{i \in d V(\boldsymbol{x}) ; \Delta} e x_{i Q}(t, \boldsymbol{x}) Q_{i}(t, \boldsymbol{x}) \\
E T_{S V}(t, x)=E x_{S V}(t, \boldsymbol{x}) S V(t, \boldsymbol{x})=\sum_{i \in d V(\boldsymbol{x}) ; \Delta} e x_{i S V}(t, \boldsymbol{x}) S V_{i}(t, \boldsymbol{x}) \\
\boldsymbol{E} \boldsymbol{x}(t, \boldsymbol{x})=\left(E x_{Q}(t, \boldsymbol{x}) ; E x_{S V}(t, \boldsymbol{x})\right)
\end{gathered}
$$

Expected transactions $\boldsymbol{E T}(t, \boldsymbol{x})$ (4.3) and transactions $\boldsymbol{T r}(t, \boldsymbol{x})$ (2.1) define aggregate expectations $\boldsymbol{E} \boldsymbol{x}(t, \boldsymbol{x})(4.3 ; 4.4 .3)$ of e-particles with coordinates $\boldsymbol{x}$ in a unit $d V(t, \boldsymbol{x})$ during time $\Delta$. Relations (4.3; 4.4.1-4.4.3) describe transition from description of expectations $\boldsymbol{e x}_{i}(t, \boldsymbol{x})$ of separate agents to description of expectations $\boldsymbol{E x}(t, \boldsymbol{x})$ as functions of point $\boldsymbol{x}$ on e-space. To derive self-consistent equations that describe relations between transactions $\operatorname{Tr}(t, \boldsymbol{x})$ and expectations $\boldsymbol{E} \boldsymbol{x}(t, \boldsymbol{x})$ one should derive equations on expectations. To do that let's use expected transactions $\boldsymbol{E T}(t, \boldsymbol{x})$ (4.3) and derive economic equations for $\boldsymbol{E T}(t, \boldsymbol{x})$ similar to equations (3.4-3.6.2). Let's use relations $(2.3-2.5)$ to define "impulses" $\boldsymbol{\Pi}(t, \boldsymbol{x})$ of expected transactions $\boldsymbol{E T}(t, \boldsymbol{x})$. Economic meaning of "impulses" $\boldsymbol{\Pi}(t, \boldsymbol{x})$ is similar to transactions impulses. Impulses $\boldsymbol{\Pi}_{i}(t, \boldsymbol{x})$ of e-particle $i$ at point describe flow of expected transaction $\boldsymbol{e} \boldsymbol{t}_{i}(t, \boldsymbol{x})=\boldsymbol{e} \boldsymbol{x}_{i}(t, \boldsymbol{x}) \boldsymbol{t r}_{i}(t, \boldsymbol{x})$ induced by motion of e-particle $i$ on e-space with velocity $\boldsymbol{v}_{i}=\left(v_{1}, \ldots v_{n}\right)$. Let's define impulses $\boldsymbol{\Pi}_{i}(t, \boldsymbol{x})$ as:

$$
\begin{gathered}
\Pi_{i}(t, \boldsymbol{x})=\boldsymbol{e} \boldsymbol{t}_{\boldsymbol{i}}(t, \boldsymbol{x}) \boldsymbol{v}_{\boldsymbol{i}}(t, \boldsymbol{x})=\left(e t_{i Q}(t, \boldsymbol{x}) \boldsymbol{v}_{i Q}(t, \boldsymbol{x}) ; e t_{i S V}(t, \boldsymbol{x}) \boldsymbol{v}_{i S V}(t, \boldsymbol{x})\right) \\
\boldsymbol{e t}_{\boldsymbol{i}}(t, \boldsymbol{x}) \boldsymbol{v}_{\boldsymbol{i}}(t, \boldsymbol{x})=\boldsymbol{e} \boldsymbol{x}_{\boldsymbol{i}}(t, \boldsymbol{x}) \boldsymbol{t r}_{\boldsymbol{i}}(t, \boldsymbol{x}) \boldsymbol{v}_{\boldsymbol{i}}(t, \boldsymbol{x}) \\
e t_{i Q}(t, \boldsymbol{x}) \boldsymbol{v}_{i Q}(t, \boldsymbol{x})=e x_{i Q}(t, \boldsymbol{x}) Q_{i}(t, \boldsymbol{x}) \boldsymbol{v}_{\boldsymbol{i Q}}(t, \boldsymbol{x})
\end{gathered}
$$




$$
\begin{gathered}
e t_{i S V}(t, \boldsymbol{x}) \boldsymbol{v}_{i S V}(t, \boldsymbol{x})=e x_{i S V}(t, \boldsymbol{x}) S V_{i}(t, \boldsymbol{x}) \boldsymbol{v}_{\boldsymbol{i S V}}(t, \boldsymbol{x}) \\
\boldsymbol{v}_{\boldsymbol{i}}(t, \boldsymbol{x})=\left(\boldsymbol{v}_{\boldsymbol{i} \boldsymbol{Q}}(t, \boldsymbol{x}) ; \boldsymbol{v}_{\boldsymbol{i S V}}(t, \boldsymbol{x})\right)
\end{gathered}
$$

Let's aggregate $\boldsymbol{\Pi}_{i}(t, \boldsymbol{x})$ in a unit volume $d V(\boldsymbol{x})$ and time term $\Delta$ similar to (2.5.1-2.5.5):

$$
\begin{gathered}
\boldsymbol{\Pi}(t, \boldsymbol{x})=\left(\boldsymbol{\Pi}_{Q}(t, \boldsymbol{x}) ; \boldsymbol{\Pi}_{S V}(t, \boldsymbol{x})\right)=\sum_{i \in d V(\boldsymbol{x}) ; \Delta} \boldsymbol{\Pi}_{i}(t, \boldsymbol{x}) \\
\boldsymbol{\Pi}_{Q}(t, \boldsymbol{x})=E x_{Q}(t, \boldsymbol{x}) Q(t, \boldsymbol{x}) \boldsymbol{u}_{Q}(t, \boldsymbol{x})=\sum_{i \in d V(\boldsymbol{x}) ; \Delta} e x_{i Q} Q_{i}(t, \boldsymbol{x}) \boldsymbol{v}_{i}(t, \boldsymbol{x}) \\
\boldsymbol{\Pi}_{S V}(t, \boldsymbol{x})=E x_{S V}(t, \boldsymbol{x}) S V(t, \boldsymbol{x}) \boldsymbol{u}_{S V}(t, \boldsymbol{x})=\sum_{i \in d V(\boldsymbol{x}) ; \Delta} \operatorname{ex}_{i S V} S V_{i}(t, \boldsymbol{x}) \boldsymbol{v}_{i}(t, \boldsymbol{x}) \\
\boldsymbol{u}(t, \boldsymbol{x})=\left(\boldsymbol{u}_{Q}(t, \boldsymbol{x}) ; \boldsymbol{u}_{S V}(t, \boldsymbol{x})\right)
\end{gathered}
$$

Relations (4.3-4.4.3) and (4.5.1-4.5.5) show that expectations $\boldsymbol{E} \boldsymbol{x}(t, \boldsymbol{x})$ as medium on e-space has velocities $\boldsymbol{u}(t, \boldsymbol{x})$ (4.5.5) that can be different from velocities $\boldsymbol{v}(t, \boldsymbol{x})$ of transactions (2.5.5). Economic equations on expected transactions $\boldsymbol{E T}(t, \boldsymbol{x}), \boldsymbol{\Pi}(t, \boldsymbol{x})$ and $\boldsymbol{u}(t, \boldsymbol{x})$ determined by (4.5.14.5.6) take form similar to equations (3.4-3.6):

$$
\begin{gathered}
\frac{\partial E \boldsymbol{T}}{\partial t}+\nabla \cdot(\boldsymbol{u} \boldsymbol{E T})=\boldsymbol{G}_{1} ; \boldsymbol{G}_{1}=\left(G_{1 Q} ; G_{1 S V}\right) \\
\frac{\partial E T_{Q}}{\partial t}+\nabla \cdot\left(\boldsymbol{u}_{Q} E T_{Q}\right)=G_{1 Q} ; \frac{\partial E T_{S V}}{\partial t}+\nabla \cdot\left(\boldsymbol{u}_{S V} E T_{S V}\right)=G_{1 S V} \\
\frac{\partial \boldsymbol{\Pi}}{\partial t}+\nabla \cdot(\boldsymbol{u} \boldsymbol{\Pi})=\boldsymbol{G}_{2} ; \boldsymbol{G}_{2}=\left(\boldsymbol{G}_{2 Q} ; \boldsymbol{G}_{2 S V}\right) \\
\frac{\partial \boldsymbol{\Pi}_{Q}}{\partial t}+\nabla \cdot\left(\boldsymbol{u}_{Q} \boldsymbol{\Pi}_{Q}\right)=\boldsymbol{G}_{2 Q} ; \frac{\partial \boldsymbol{\Pi}_{S V}}{\partial t}+\nabla \cdot\left(\boldsymbol{u}_{S V} \boldsymbol{\Pi}_{S V}\right)=\boldsymbol{G}_{2 S V}
\end{gathered}
$$

Economic equations (5.1-5.4) are similar to equations (3.4-3.6) and describe evolution of expected transactions $\boldsymbol{E} \boldsymbol{T}(t, \boldsymbol{x}), \boldsymbol{\Pi}(t, \boldsymbol{x})$ under action of factors $\boldsymbol{G}_{\boldsymbol{1}}$ and $\boldsymbol{G}_{2}$.

Equations (3.4-3.6) and equations (5.1-5-4) allow derive equations on expectations $\boldsymbol{E} \boldsymbol{x}(t, \boldsymbol{x})$ :

$$
\begin{gathered}
\frac{\partial \boldsymbol{E} \boldsymbol{T}}{\partial t}+\nabla \cdot(\boldsymbol{u E T})=\boldsymbol{G}_{1} ; \boldsymbol{G}_{1}=\left(G_{1 Q} ; G_{1 S V}\right) \\
\frac{\partial}{\partial t} \boldsymbol{E} \boldsymbol{x} \boldsymbol{T r}+\nabla \cdot(\boldsymbol{u} \boldsymbol{E} \boldsymbol{x} \boldsymbol{T r})=\boldsymbol{G}_{1} ; \boldsymbol{G}_{1}=\left(G_{1 Q} ; G_{1 S V}\right)
\end{gathered}
$$

\section{Simple model relations between Transactions and Expectations}

To model mutual impact of expectations $\boldsymbol{E x}(t, \boldsymbol{x})$ on transactions $\boldsymbol{T r}(t, \boldsymbol{x})$ and vise versa let's assume that factors $\boldsymbol{F}_{1}$ and $\boldsymbol{F}_{2}$ depend on expected transactions $\boldsymbol{E T}(t, \boldsymbol{x})$ (4.3) and their impulses $\boldsymbol{\Pi}(t, \boldsymbol{x})$ and velocities $\boldsymbol{u}(t, \boldsymbol{x})$ or their linear operators and factors $\boldsymbol{G}_{\boldsymbol{1}}$ and $\boldsymbol{G}_{\boldsymbol{2}}$ have similar dependence on $\boldsymbol{T r}(t, \boldsymbol{x}), \boldsymbol{P}(t, \boldsymbol{x})$ and $\boldsymbol{v}(t, \boldsymbol{x})$. Such approximation permits describe selfconsistent system of equations (3.4-3.6.3) and (5.1-5.4) that model mutual relations between transactions $\boldsymbol{T r}(t, \boldsymbol{x})$ and expected transactions $\boldsymbol{E} \boldsymbol{T}(t, \boldsymbol{x})$. Our approach models mutual dependence between transactions and expectations and shows possible hidden origin for price chaotic fluctuations. Nevertheless we simplify relations between Transactions and 
Expectations the system of economic equations (3.4-3.6.3) and (5.1-5.4) remains sufficiently complex and we propose further simplifications.

Let's propose that we know slow mean evolution of transactions $\boldsymbol{T r}(t, \boldsymbol{x})$ and expected transactions $\boldsymbol{E T}(t, \boldsymbol{x})$. Let's describe evolution of small disturbances near mean values. Let's present transactions $\boldsymbol{T} \boldsymbol{r}(t, \boldsymbol{x})$ and expectations $\boldsymbol{E} \boldsymbol{T}(t, \boldsymbol{x})$ as:

$$
\boldsymbol{T r}(t, \boldsymbol{x})=\boldsymbol{T r}_{\mathbf{0}}(t, \boldsymbol{x})+\boldsymbol{t r}(t, \boldsymbol{x}) ; \boldsymbol{E T}(t, \boldsymbol{x})=\boldsymbol{E T}_{\mathbf{0}}(t, \boldsymbol{x})+\boldsymbol{e t}(t, \boldsymbol{x})
$$

Let's neglect time dependence of mean Transactions $\boldsymbol{T r}_{\boldsymbol{0}}(t, \boldsymbol{x})$ and expected transactions $\boldsymbol{E} \boldsymbol{T}_{\boldsymbol{0}}(t, \boldsymbol{x})$ to compare with time dependence of disturbances $\boldsymbol{t r}(t, \boldsymbol{x})$ and $\boldsymbol{e t}(t, \boldsymbol{x})$ respectively. Relations (6.1) describe transactions $\boldsymbol{T r}(t, \boldsymbol{x})$ as sum of mean transactions $\boldsymbol{T r}_{\boldsymbol{0}}(t, \boldsymbol{x})$ and small disturbances $\boldsymbol{t r}(t, \boldsymbol{x})$ and we use same representation for expected transactions $\boldsymbol{E T}(t, \boldsymbol{x})$. In Appendix A we present simple model with linear dependence between disturbances of transactions $\boldsymbol{t r}(t, \boldsymbol{x})$ and expected transactions $\boldsymbol{e t}(t, \boldsymbol{x})$. Equations on disturbances $\boldsymbol{t r}(t, \boldsymbol{x})$ (A.1.3) and $\boldsymbol{e t}(t, \boldsymbol{x})$ (A.2.3) for the assumptions (A.3.1; 3.2) take form (A.4.1-4.2) and (A.4.4):

$$
\begin{aligned}
& \frac{d}{d t} q(t)=\alpha_{Q} e t_{q}(t) ; \frac{d}{d t} s v(t)=\alpha_{S V} e t_{s v}(t) \\
& \frac{d}{d t} e t_{q}(t)=\beta_{Q} q(t) ; \frac{d}{d t} e t_{S V}(t)=\beta_{S V} s v(t)
\end{aligned}
$$

Equations (6.1-6.3) or (A.4.1-A.4.5) define dimensionless price disturbances $\pi(t)$ (A.5.1A.5.3):

$$
\pi(t)=c \sin \omega_{S V} t-d \sin \omega_{Q} t ; c \ll 1 ; d \ll 1
$$

Relations (6.4) present simplest harmonic oscillations of price disturbances $\pi(t)$. It seems that such simple approximation can't describe chaotic fluctuations of price disturbances. Meanwhile we propose that even simple approximation can be origin for development of more complex models that may describe chaotic price fluctuations.

\section{Price fluctuations, Expectations and Lorenz attractor}

In this Section we show that disturbances of trading volume $q(t)$, price $\pi(t)$ and Expectations $e x_{q}(t)$ and $e x_{s v}(t)$ can become origin for deterministic chaotic fluctuations due to parallels to Lorenz attractor. Let's start with equations (A.1.1) on transactions $\boldsymbol{T} \boldsymbol{r}(t)$ (A.1.3) and equations (A.2.1) on expected transactions $\boldsymbol{E T}(t)$ (A.2.2; A.2.3) and obtain equations on disturbances of trading volume $q(t)$ (A.1.3), price $\pi(t)$ (A.5.1) and disturbances of Expectations $e x_{q}(t)$ and $e x_{s v}(t)(4.3-4.4 .3)$ that we denote as:

$$
E x_{Q}(t)=E x_{Q 0}(t)+e x_{q}(t) ; E x_{S V}(t)=E x_{S V 0}(t)+e x_{S v}(t)
$$

Equations (A.1.1) allow derive equations on volume $Q(t)$, price $p(t)$, and (A.2.1) on expectations $E x_{Q}(t)$ and $E x_{S V}(t)$. Equations on volume $Q(t)$ take form 


$$
\frac{d Q(t)}{d t}=F_{1 Q}(t)
$$

Equations on price $p(t)$ follow from $(2.2)$ and (A.1.1; 7.2):

$$
\frac{d S V(t)}{d t}=\frac{d p(t) Q(t)}{d t}=Q(t) \frac{d p(t)}{d t}+p(t) F_{1 Q}(t)=F_{1 S V}(t)
$$

Equations on expectations $E x_{Q}(t)$ and $E x_{S V}(t)$ result from (4.3-4.4.3) and (A.2.1):

$$
\begin{gathered}
\frac{d E T_{Q}}{d t}=Q(t) \frac{d E x_{Q}}{d t}+E x_{Q}(t) F_{1 Q}(t)=G_{1 Q}(t) \\
\frac{d E T_{S V}}{d t}=p(t) Q(t) \frac{d E x_{S V}(t)}{d t}+E x_{S V}(t) F_{1 S V}(t)=G_{1 S V}(t)
\end{gathered}
$$

To derive equations on disturbances $q(t), \pi(t), e x_{q}(t), e x_{s v}(t)($ A.1.3;A.5.1;7.1) let's take righthand factors in (7.2 -7.4) $F_{1 Q}(t), F_{1 S V}(t), G_{1 Q}(t), G_{I S V}(t)$ as (A.1.4; A.2.4). Let's assume that mean values $Q_{0}(t), p_{0}(t), E x_{Q 0}(t)$ and $E x_{S V 0}(t)$ are slow to compare with variability of disturbances $q(t), \pi(t), e x_{q}(t), e x_{s v}(t)$ and neglect all derivations by time for mean values $Q_{0}(t)$, $p_{0}(t), E x_{Q O}(t)$ and $E x_{S V O}(t)$ to compare with derivations of disturbances. Let's take all slow mean variables as constants and neglect all nonlinear factors for disturbances. Hence obtain equations on dimensionless disturbances $q(t), \pi(t), e x_{q}(t), e x_{s v}(t)$ :

$$
\begin{aligned}
& Q_{0} \frac{d q(t)}{d t}=f_{1 q}(t) \\
& Q_{0} p_{0} \frac{d \pi(t)}{d t}+p_{0} f_{1 q}(t)+p_{0} \pi(t) F_{1 Q 0}=f_{1 s v}(t) \\
& Q_{0} \frac{d}{d t} e x_{q}(t)+E x_{Q 0} f_{1 q}(t)+e x_{q}(t) F_{1 Q 0}=g_{1 q}(t) \\
& Q_{0} p_{0} \frac{d}{d t} e x_{s v}(t)+E x_{S V 0} f_{1 s v}(t)+e x_{s v}(t) F_{1 S V 0}=g_{1 s v}(t)
\end{aligned}
$$

Equations (8.1-8.4) define relations on dimensionless disturbances of trading volume $q(t)$, price disturbances $\pi(t)$ and disturbances of expectations of trading volume $e x_{q}(t)$ and stock value $e x_{s v}(t)$. Equations (8.1-8.4) with right-hand side factors $f_{1 q}(t), f_{1 s v}(t), g_{1 q}(t), g_{1 s v}(t)$ have form of dynamical system equations. During last decades such systems established new research area that describes deterministic chaotic behavior of coupled oscillators, attractors, and non-linear dynamical systems. We note only small part of studies on chaotic dynamics (Lorenz, 1963; Andronov, Vitt and Khaikin, 1966; Mandelbrot, 1977; Takens, 1981; Thompson and Stewart, 1986; Sagdeev, Usikov and Zaslavsky, 1988; Nicolis and Prigogin, 1989; Guckenheimer, Holmes, 1990; Bullard and Butler, 1991; Neimark and Landa, 1992; Brock and Hommes, 1997; Ott, 2002; Schuster and Just, 2005; Goldstein, J., 2011).

Below we show that equations on dimensionless disturbances of trading volume $q(t)$, price $\pi(t)$ and expectations disturbances $e x_{q}(t)$ can take form of Lorenz attractor. For convenience let's denote

$$
x=q(t) ; y=\pi(t) ; z=\operatorname{ex}_{q}(t)
$$


We derive equations (8.1-8.4) in the assumption that dimensionless disturbances $q(t), \pi(t)$, $e x_{q}(t)$ are small:

$$
x=q(t)<1 ; y=\pi(t)<1 ; z=e x_{q}(t)<1
$$

Let's determine scales of small disturbances (9.1-9.2) as follows. Let's model price disturbances $\pi(t)$ in the assumption that mean price $p_{0}(t)$ can be regarded as constant. Intraday price fluctuations of Stocks at Exchange are usually don't exceed 5\% of mean price. Let's describe disturbances $(9.1 ; 9.2)$ in the assumption that price fluctuations $\pi(t)$ don't exceed $5 \%$ during time term T. Let's take trading volume fluctuations $q(t)$ and expectations $e x_{q}(t)$ less than one. To develop parallels between equations (8.1-8.4) that should describe small disturbances during time term $\mathrm{T}$ and Lorenz equations (10.1-10.3). Let's underline that trajectories of Lorenz attractor (Lorenz, 1963)

$$
\begin{aligned}
& \frac{d x}{d t}=\sigma(y-x) \\
& \frac{d y}{d t}=-y-x z+r x \\
& \frac{d z}{d t}=x y-b z
\end{aligned}
$$

don't describe small fluctuations near zero. More over, mean values of variables $x, y, z$ during time term T don't equal zero. Let's define mean values $X(T), Y(T), Z(T)$ as

$$
X(T)=\frac{1}{T} \int_{0}^{T} d t x(t) ; Y(T)=\frac{1}{T} \int_{0}^{T} d t y(t) ; Z(T)=\frac{1}{T} \int_{0}^{T} d t z(t)
$$

and redefine variables as

$$
x \rightarrow x^{\prime}+X ; y \rightarrow y^{\prime}+Y ; z \rightarrow z^{\prime}+Z
$$

Then variables $x^{\prime}, y^{\prime}, z^{\prime}$ for equations (10.1-10.3) will have mean values equal zero. Now let's rescale (10.1-10.3) so they can describe variables due to (9.2). To do that let's change scales of Lorenz equations as follows. Let denote amplitudes along axes $x, y, z$ as $\min L, N, M$ that obey:

$$
|x(t)| \leq L ;|y(t)| \leq 5 \% N ;|z(t)| \leq M, t \leq T
$$

Then scale transformation (10.7) for time term $t<T$

$$
\mathrm{x} \rightarrow L x ; \quad y \rightarrow N y ; \quad z \rightarrow M z
$$

defines dimensionless disturbances of price $\pi(t)=y$ with amplitudes less then $5 \%$ and trading volume $q(t)$, and expectations $e x_{q}(t)$ fluctuations with amplitudes less then 1 . Transition (10.5) and (10.7) transforms Lorenz equations (10.1-10.3) as follows:

$$
\begin{gathered}
\frac{d x}{d t}=\sigma\left(\frac{N}{L} y-x\right)+\frac{\sigma}{L}(Y-X) \\
\frac{d y}{d t}=\frac{L}{N}(r-Z) x-y-\frac{X M}{N} z-\frac{L M}{N} x Z+\frac{1}{N}(r X-Y-X Z)
\end{gathered}
$$




$$
\frac{d z}{d t}=\frac{Y L}{M} x+\frac{X N}{M} y-b z+\frac{L N}{M} x y+\frac{1}{M}(X Y-b Z)
$$

Here constants $X, Y, Z$ define mean values $(10.4 ; 10.5)$ for $t<T$ and constants $L, N, M$ - define amplitude scales $(10.6 ; 10.7)$. Hence dimensionless variables $x, y, z$ for modified Lorenz equations (10.8-10.10) have zero mean values and amplitudes of $x$ and $z$ are less than 1 and amplitude of $y$ is less than $5 \%$ for time term $t<T$. If $T$ is large enough than it's valid for different realizations of (10.8-10.10). It is easy to verify that relations (11.1-11.3):

$$
\begin{gathered}
f_{1 q}(t)=Q_{0} \sigma\left[\left(\frac{N}{L} y-x\right)+\frac{1}{L}(Y-X)\right] \\
f_{1 s v}(t)=Q_{0} p_{0}\left\{\left[\frac{L}{N}(r-Z)-\sigma\right] x+\left[\left(\sigma \frac{N}{L}+\frac{F_{1 Q 0}}{Q_{0}}\right)-1\right] y-\frac{X M}{N} z-\frac{L M}{N} x z+\frac{1}{N}(r X-Y-\right. \\
\left.X Z)+\frac{\sigma}{L}(Y-X)\right\} \\
g_{1 q}(t)=Q_{0}\left[\left(\frac{Y L}{M}-E x_{Q 0} \sigma\right) x+\left(\frac{X N}{M}+E x_{Q 0} \sigma \frac{N}{L}\right) y+\left(\frac{F_{1 Q 0}}{Q_{0}}-b\right) z+\frac{L N}{M} x y+\right. \\
\left.E x_{Q 0} \frac{\sigma}{L}(Y-X)+\frac{1}{M}(X Y-b Z)\right]
\end{gathered}
$$

present equations (8.1-8.3) as modified Lorenz equations (10.8-10.10). Let's underline that all constants those define scales of trading volume $Q_{0}$, price $p_{0}$ and expectations $E x_{Q 0}, E x_{S V O}$ remain as free parameters. Hence (10.1-10.3) and modified Lorenz equations (10.8-10.10) are valid for different $Q_{0}, p_{0}, E x_{Q 0}$ and $E x_{S V O}$.

Economic meaning of Lorenz equations (10.8-10.10) for disturbances of trading volume $q(t)$, price $\pi(t)$ and expectations $e x_{q}(t)$ can be treated as follows. Equation (10.8) states that time derivative of trading volume disturbances $x=q(t)$ is positively proportional to price disturbances $y=\pi(t)$ and negative to trading volume $x=q(t)$. Indeed, growth of price $y=\pi(t)$ causes growth of trading volume $q(t)$. As well high trading volumes induce decline of further growth of trading volumes. Equation (10.9) states that time derivative of price $y=\pi(t)$ rise up with growth of trading volumes $x=q(t)$ if $r$ is more then amplitude of expectations $Z$. Otherwise time derivative of price $y=\pi(t)$ has negative responses on trading volumes $x=q(t)$, prices $y=\pi(t)$, expectations $z=e x_{q}(t)$ and on product of trading volumes $x=q(t)$ and expectations of trading volumes $z=e x_{q}(t)$. Indeed, growth of trading volume $x=q(t)$ should cause rise of price $y=\pi(t)$ if amplitude of expectations of trading volumes is relatively small. Otherwise, high trading volume expectations will decline price time variable. As well rise of price $y=\pi(t)$ should induce further decline of price derivation $d y / d t$. High product of trading volumes $x=q(t)$ and expectations of trading volumes $z=e x_{q}(t)$ indicates possible growth of trading volumes in future and that should reduce price derivation $d y / d t$. Equation (10.10) on expectations of trading volumes $z=e x_{q}(t)$ states that time derivation of $z=e x_{q}(t)$ has negative 
response on high expectations $z=e x_{q}(t)$ and positive response on trading volume $x=q(t)$, price $y=\pi(t)$ and product of trading volume $x=q(t)$ and prices $y=\pi(t)$. Indeed, high values of product of trading volume and prices $x y=q(t) \pi(t)$ should cause positive growth of expectations of trading volumes $z=e x_{q}(t)$. On the other hand high values of expectations of trading volumes $z=e x_{q}(t)$ decline its time derivative due to expected saturation of market. Thus modified Lorenz equations (10.8-10.10) have reasonable economic meaning for small disturbances of trading volume $x=q(t)$, price $y=\pi(t)$ and trading volume expectations $z=e x_{q}(t)$. Modified Lorenz equation (10.8-10.10) may describe small fluctuations of trading volume $|q(t)|<1$, price $|\pi(t)|<5 \%$ and expectations $\left|e x_{q}(t)\right|<1$ for time term $t<T$. Description of expectations $e x_{s v}(t)$ due to equations (8.4) require assumptions on function $g_{1 s v}(t)$. Further description of dimensionless disturbances $x=q(t), y=\pi(t)$ and $z=e x_{q}(t)$ for $t>T$ may require taking into account changes of mean values of trade volumes $Q_{0}(t)$, price $p_{0}(t)$ and Expectations $E x_{Q O}(t)$ and other factors that may impact price fluctuations. These effects should be studied in upcoming papers.

\section{Conclusions}

We reduce our research by simple model relations between transactions and expectations. It seems interesting that general considerations about agents distributions on economic space allow derive equations (8.1-8.4) on disturbances of trading volumes $q(t)$, price $\pi(t)$ and expectations disturbances $e x_{q}(t)$ and $e x_{s v}(t)$. As we show that equations (8.2-8.4) can take form of Lorenz attractor. Description of price fluctuations by strange attractor model indicated possible deterministic origin for chaotic price disturbances. That is consistent with numerous studies that proposed deterministic models for the random price origin (Barnett and Chen, 1988; Brock and Sayers, 1988; Bullard and Butler, 1991; Brock and Hommes, 1997; 1998).

Lorenz attractor and similar non-linear dynamical systems are studied during last fifty years. We are not going to repeat here known results on Lorenz attractor and refer to numerous studies (Lorenz, 1963; Takens, 1981; Thompson and Stewart, 1986; Sagdeev et.al, 1988; Guckenheimer and Holmes, 1990; Ott, 2002; Anishchenko et.al, 2003; Schuster and Just, 2005; Loskutov, 2010; Goldstein, 201; Broer and Takens, 2011). Lorenz model (10.1-10.3) for price and expectations disturbances gives new look on possible nature of price and return fluctuations and that issue should be studied further. Fluctuations of asset prices due to Lorenz attractor equations can have impact on forecasting macro financial variables and on assessments of option pricing. It is clear that equations (8.1-8.4) and (10.1-10.3) describe 
only part of reasons for chaotic price fluctuations. Various random shocks and impact of numerous financial variables that were neglected in our treatment for sure play important role for price and returns fluctuations. Equations (8.1-8.4) and their extensions can be used for modeling properties of price and trading volume fluctuations by strange attractors and allow apply general dynamical systems methods (Lorenz, 1963; Guckenheimer and Holmes, 1990; Neimark and Landa, 1992; Ott, 2002; Anishchenko et.al, 2003; Broer and Takens, 2011; Goldstein, 2011). We regard our result and representation of price fluctuations within Lorenz attractor as only a possible case for description of chaotic processes in financial markets. A lot of further studies are required to verify above approximations with observed data. Let's repeat that our approach to description of economic and financial transactions, expectations and price fluctuations don't need any assumptions and methods of general equilibrium and regard them as unnecessary.

We propose that econometrics can provide sufficient data for assessment of risk ratings of economic agents and modeling their economic and financial variables and transactions on economic space. Distributions of agents by their risk ratings on economic space define transactions and expectations as functions of coordinates. Expectations play crucial role for evaluating transactions. We simplified relations between transactions and expectations to study their mutual impact in self-consistent manner. We hope that our model and results can be useful for further studies of expectations, price fluctuations and for application of modern dynamical systems methods for modeling macroeconomics and macro finance. 
Appendix A

\section{Simple solution for price disturbances}

Let's start with equations (3.4) on transactions $\boldsymbol{T} \boldsymbol{r}(t, \boldsymbol{x})$ and derive equations on transactions $\boldsymbol{T r}(t)$ as function of time only. To do that let's take integral of (3.4) by $d \boldsymbol{x}$ over economic domain (1.2):

$$
\begin{gathered}
\boldsymbol{T} \boldsymbol{T}(t)=\int d x \boldsymbol{T r}(t, \boldsymbol{x}) ; \boldsymbol{E T}(t)=\int d x \boldsymbol{E T}(t, \boldsymbol{x}) \\
\int d x \frac{\partial}{\partial t} \boldsymbol{T r}(t, \boldsymbol{x})+\int d x \nabla \cdot(\boldsymbol{v} \boldsymbol{T r})=\int d x \boldsymbol{F}_{1}(t, \boldsymbol{x}) \\
\int d x \frac{\partial}{\partial t} \boldsymbol{T r}(t, \boldsymbol{x})=\frac{d}{d t} \int d x \boldsymbol{T} \boldsymbol{T}(t, \boldsymbol{x})=\frac{d}{d t} \boldsymbol{T r}(t) ; \int d x \boldsymbol{F}_{1}(t, \boldsymbol{x})=\boldsymbol{F}_{1}(t)
\end{gathered}
$$

As we mentioned above Divergence theorem (Strauss 2008, p.179) states that surface integral of flux $\boldsymbol{v} \boldsymbol{T r}$ through surface of a unit volume equals volume integral of divergence $\boldsymbol{v} \boldsymbol{T r}$. Economic transactions are defined on economic domain (1.2) and are equal zero out of economic domain. Hence integral by divergence over economic domain equals surface integral of flux $\boldsymbol{v} \boldsymbol{T r}$ through surface of economic domain and equals zero as no fluxes out or in economic domain exist. The same statement valid for integral of flux $\boldsymbol{u E T}$ through surface of economic domain:

$$
\int d x \nabla \cdot(\boldsymbol{v T r})=0 ; \int d x \nabla \cdot(\boldsymbol{u T r})=0
$$

thus equations $(3.4,3.5)$ take form:

$$
\frac{d}{d t} \boldsymbol{T} \boldsymbol{r}(t)=\boldsymbol{F}_{1}(t) ; \frac{d Q(t)}{d t}=F_{1 Q}(t) ; \frac{d S V(t)}{d t}=F_{1 S V}(t)
$$

The same reasons are valid to equations on expectations $(5.1 ; 5.2)$. Due to $(6.1)$ transactions $\boldsymbol{T R}(t)$ take form

$$
\boldsymbol{T r}(t)=\boldsymbol{T r}_{0}(t)+\boldsymbol{t r}(t) ; \boldsymbol{T r}_{0}(t)=\int d x \boldsymbol{T r}_{0}(t, \boldsymbol{x}) ; \boldsymbol{t r}(t)=\int d x \operatorname{tr}(t, \boldsymbol{x})
$$

Due to (2.6)

$$
\begin{array}{r}
\boldsymbol{T r}_{0}(t)=\left(Q_{o}(t) ; S V_{0}(t)\right) ; \boldsymbol{t r}(t)=(q(t) ; s v(t)) \\
Q(t)=Q_{0}(t)+q(t) ; S V(t)=p(t) Q(t)=S V_{0}(t)+s v(t)
\end{array}
$$

Let's assume that factor $\boldsymbol{F}_{1}(t)$ can be presented as mean part $\boldsymbol{F}_{10}(t)$ and disturbances $\boldsymbol{f}_{1}(t)$ as:

$$
\boldsymbol{F}_{1}(t)=\boldsymbol{F}_{10}(t)+\boldsymbol{f}_{1}(t) ; F_{1 Q}(t)=F_{10 Q}(t)+f_{1 q}(t) ; \quad F_{1 S V}(t)=F_{10 S V}(t)+f_{1 s v}(t)
$$

Equation (A.1.1) on disturbances $\operatorname{tr}(t)$ takes form:

$$
\frac{d}{d t} \operatorname{tr}(t)=\boldsymbol{f}_{1}(t) ; \frac{d q(t)}{d t}=f_{1 q}(t) ; \frac{d s v(t)}{d t}=f_{1 s v}(t)
$$


Here $\boldsymbol{f}_{1}(t)$ describes action of disturbances factors only and we propose that $\boldsymbol{f}_{1}(t)$ depends on disturbances of expectations $\boldsymbol{e t}(t)$ and neglect slow action of mean expectations $\boldsymbol{E T}_{\boldsymbol{0}}(t)$. The same assumptions are valid for expectations and their disturbances.

$$
\begin{gathered}
\frac{d \boldsymbol{E} \boldsymbol{T}(t)}{d t}=\boldsymbol{G}_{1}(t) ; \frac{d E T_{Q}}{d t}=G_{1 Q}(t) ; \frac{d E T_{S V}(t)}{d t}=G_{1 S V}(t) \\
\boldsymbol{E} \boldsymbol{T}(t)=\boldsymbol{E} \boldsymbol{T}_{0}(t)+\boldsymbol{e t}(t) ; \boldsymbol{E} \boldsymbol{T}_{0}(t)=\int d x \boldsymbol{E T}_{0}(t, \boldsymbol{x}) ; \boldsymbol{e t}(t)=\int d x \boldsymbol{e t}(t, \boldsymbol{x}) \\
E T_{Q}(t)=E T_{Q 0}(t)+e t_{q}(t) ; E T_{S V}(t)=E T_{S V 0}(t)+e t_{S v}(t)
\end{gathered}
$$

We propose that factor $\boldsymbol{G}_{l}(t)$ can be presented as mean part $\boldsymbol{G}_{10}(t)$ and disturbances $\boldsymbol{g}_{l}(t)$ similar to (A.1.4) :

$$
\begin{gathered}
\boldsymbol{G}_{1}(t)=\boldsymbol{G}_{10}(t)+\boldsymbol{g}_{\mathbf{1}}(t) \\
G_{1 Q}(t)=G_{10 Q}(t)+g_{1 q}(t) ; \quad G_{1 S V}(t)=G_{10 S V}(t)+g_{1 s v}(t)
\end{gathered}
$$

and due to (A.2.1) equations on expectations $\boldsymbol{e t}(t)$ disturbances take form:

$$
\frac{d}{d t} \boldsymbol{e t}(t)=\boldsymbol{g}_{1}(t) ; \frac{d}{d t} \boldsymbol{e} t_{q}(t)=g_{1 q}(t) ; \frac{d}{d t} e t_{s v}(t)=g_{1 s v}(t)
$$

To simplify relations between transactions and expectations let's assume that factor $\boldsymbol{f}_{l}$ linear depends on expectations disturbances and equations (A.1.1) on disturbances $\boldsymbol{b}(t)$ take form:

$$
\frac{d}{d t} \boldsymbol{t r}(t)=\boldsymbol{f}_{1}(t)=\boldsymbol{\alpha} \boldsymbol{e t}(t)=\left(\alpha_{Q} e t_{q}(t) ; \alpha_{S V} e t_{s v}(t)\right) ; \boldsymbol{\alpha}=\left(\alpha_{Q} ; \alpha_{S V}\right)
$$

Coefficients $\alpha_{Q}$ and $\alpha_{S V}$ are constant. Let's make same assumptions for equations (A.2.5) and take equations on expectations disturbances $\boldsymbol{e t}(t)$ in the form similar to (A.3.1):

$$
\begin{gathered}
\frac{d}{d t} \boldsymbol{e t}(t)=\boldsymbol{g}_{1}(t)=\boldsymbol{\beta} \boldsymbol{t r}(t)=\left(\beta_{Q} q(t) ; \beta_{S V} s v(t)\right) ; \boldsymbol{\beta}=\left(\beta_{Q} ; \beta_{S V}\right) \\
\boldsymbol{f}_{1}(t)=\left(\alpha_{Q} e t_{q}(t) ; \alpha_{S V} e t_{s v}(t)\right) ; \boldsymbol{g}_{1}(t)=\left(\beta_{Q} q(t) ; \beta_{S V} s v(t)\right)
\end{gathered}
$$

Assumptions (A.3.3) describe simplest model relations between transactions and expectations and present equations (A.3.1-3.2) in a closed form. We neglect action of transactions impulses $\boldsymbol{P}(t, \boldsymbol{x})$ and expectations impulses $\boldsymbol{\Pi}(t, \boldsymbol{x})$ on evolution of transactions and expectations disturbances and reduce system of equations (3.4-3.6.3) and (5.1-5.4) to system of equations (A.3.1-3.2). For components of transactions disturbances $\operatorname{tr}(t)=(q(t) ; s v(t))$ (A.1.3) and expectations disturbances $\boldsymbol{e t}(t)=\left(e t_{q}(t) ; e t_{s v}(t)\right)(\mathrm{A} .2 .3)$ equations (A.3.1-3.2) take form:

$$
\begin{aligned}
& \frac{d}{d t} q(t)=\alpha_{Q} e t_{q}(t) ; \frac{d}{d t} s v(t)=\alpha_{S V} e t_{s v}(t) \\
& \frac{d}{d t} e_{q}(t)=\beta_{Q} q(t) ; \frac{d}{d t} e t_{s v}(t)=\beta_{S V} s v(t)
\end{aligned}
$$

For case

$$
\alpha_{Q} \beta_{Q}<0 ; \alpha_{S V} \beta_{S V}<0
$$


equations (A.4.1-4.2) describe oscillations of transactions disturbances $q(t)$ and $s v(t)$ with frequencies $\omega_{Q}$ and $\omega_{S V}$ :

$$
\begin{aligned}
& \omega_{Q}^{2}=-\alpha_{Q} \beta_{Q} ; \omega_{S V}^{2}=-\alpha_{S V} \beta_{S V} \\
& \left(\frac{d^{2}}{d t^{2}}+\omega_{Q}^{2}\right) q(t)=0 ;\left(\frac{d^{2}}{d t^{2}}+\omega_{S V}^{2}\right) s v(t)=0
\end{aligned}
$$

Transactions disturbances in simplest case take form

$$
q(t)=Q_{0}(t) c \sin \omega_{Q} t ; \operatorname{sv}(t)=S V_{0}(t) d \sin \omega_{S V} t
$$

Here $c$ and $d$ - constants that describe relative amplitudes of $q(t)$ and $s v(t)$. We propose (A.1.4) that $Q_{0}(t)$ and $S V_{0}(t)$ are slow to compare with disturbances and frequencies $\omega_{Q}$ and $\omega_{S V}$ so we treat $Q_{0}(t)$ and $S V_{0}(t)$ in relations (A.4.5) as constants and use form (A.4.5) for convenience only. Due to (2.7) and (A.1.3) price $p(t)$ of transaction (A.1.2) take form:

$$
p(t)=p_{0}(t)(1+\pi(t))
$$

$p_{0}(t)$ - is a mean price determined by ratio of slow mean stock value $\operatorname{Tr}_{O S V}(t)$ and trading volume $\operatorname{Tr}_{O Q}(t)$ components of transactions:

$$
p_{0}(t)=S V_{0}(t) / Q_{0}(t)
$$

and $\pi(t)$ - dimensionless price disturbances determined by transactions disturbances with price scale $p_{0}=p_{0}(t)$. Let's assume that mean price $p_{0}(t)$ is slow to compare with price disturbances and consider $p_{0}(t)$ as constant $p_{0}$. For the first approximation by transactions disturbances $q(t) / Q_{0}(t)$ price disturbances $\pi(t)$ take form

$$
\pi(t)=\frac{s v(t)}{S V_{0}(t)}-\frac{q(t)}{Q_{0}(t)}
$$

Simple relations (A.4.5) give for dimensionless price disturbances $\pi(t)$ :

$$
\pi(t)=d \sin \omega_{S V} t-c \sin \omega_{Q} t \quad ; c, d \ll 1
$$

Relations (A.5.3) describe simplest harmonique oscillations of dimensionless price disturbances induced by action of expectations disturbances (A.4.1-2). Market reality is much more complex, but any complex issue may have simple origin. 


\section{References}

Alejandro-Quinones, A, Bassler, K., Field, M., McCauley, J., Nicol, M., Timofeyev, I., Torok, A., Gunaratne, G.,2006. A theory of fluctuations in stock prices, Physica A, 363, 383392

Altman, E. (Ed). 2010. The Z-Metrics ${ }^{\mathrm{TM}}$ Methodology For Estimating Company Credit Ratings And Default Risk Probabilities. RiskMetrics Group Inc.

Andersen, T.G., Lund, J., 1997. Estimating continuous-time stochastic volatility models of the short-term interest rate. Journal of Econometrics 77: 343-377.

Andersen, T., Bollerslev, T., Diebold, F.X. and Ebens, H., 2001. The Distribution of Realized Stock Return Volatility, Journal of Financial Economics, 61, 43-76.

Andersen, T.G., Bollerslev, T., Peter F. Christoffersen, P.F., Diebold, F.X., 2006. Volatility and correlation forecasting. Handbook of Economic Forecasting, Ed. Elliott, G., Granger, C., Timmermann, A., North Holland, v.1, 778-878.

Andronov, A.A., Vitt, A.A., Khaikin,S.E., 1966. Theory of Oscillators. Oxford, Pergamon Press.

Anishchenko, V.S., Vadivasova, T.E., Okrokvertskhov, G.A., Strelkova, G.I., 2003.

Correlation analysis of dynamical chaos. Physica A, 325, $199-212$

Arrow, K.J., 1974. General Economic Equilibrium: Purpose, Analytic Techniques, Collective Choice. The American Economic Review, 64 (3), 253-272

Bachmann, R., Elstner, S., 2013. Firms' Optimism and Pessimism. Center for Economic Studies \& Ifo Inst., WP 4176.

Baker, A., 2007. Occam's Razor in science: a case study from biogeography. Biology and Philosophy, 22: 193-215.

Balke, N.S., Wohar, M.E. 2001. Explaining Stock Price Movements: Is There a Case for Fundamentals?, FRB Dallas, Economic and Financial Review, 3q, 22-34

Barnett, W., Chen, P., 1988. Deterministic Chaos And Fractal Attractors As Tools For Nonparametric Dynamical Econometric Inference: With An Application To The Divisia Monetary Aggregates. Math/ Comput. Modeling, 10, (4), 275-296

Bates, D.S., 1996. Jumps and stochastic volatility: exchange rate processes implicit in DM options. Review of Financial Studies, 9, 69-107.

Bayraktar, E., Horst, U., Sircar, R., 2008. Queueing Theoretic Approaches to Financial Price Fluctuations, http://arxiv.org/pdf/math/0703832

van Binsbergen, J. H., Koijen, R., 2017. The term structure of returns: Facts and theory, 
NBER WP 21234, Cambridge, MA

Blume, L.E., Easley, D., 1984. Rational Expectations Equilibrium: An Alternative Approach, Journal Of Economic Theory, 34, 116-129 (1984)

Brock, W., Sayers, C., 1988. Is the business cycle characterized by deterministic chaos? Journal of Monetary 22: 71-90.

Brock, W.A., Hommes, C.H., 1997. A Rational Routes to Randomness, Econometrica, 65 (5), 1059-1095

Brock, W.A., Hommes, C.H., 1998. Heterogeneous beliefs and routes to chaos in a simple asset pricing model. Journal of Economic Dynamics and Control, 22, 1235-1274

Broer, H., Takens, F., 2011. Dynamical Systems and Chaos. Springer, Applied Mathematical Science, v. 172

Brunnermeier, M.K., Parker, J.A., 2005. Optimal Expectations. American Economic Review, 95 (4), 1092-1118

Bullard, J.B., Butler, A., 1991. Nonlinearity and Chaos in Economic Models: Implications for Policy Decisions, WP 002B, FRB St.Louis, http://research.stlouisfed.org/wp/1991/91-002.pdf Campbell, J.Y., 1985. Stock Returns And The Term Structure, WP 1626, NBER, Cambridge Campbell, J.Y., Cochrane, J.H., 1995. By Force of Habit: A Consumption-Based Explanation of Aggregate Stock Market Behavior, NBER WP 4995, Cambridge, US

Calvet L, Fisher A. 2002. Multifractality in asset returns: theory and evidence. Review of Economics and Statistics 84: 381-406.

Carbone, A., Castelli, G., Stanley, H.E., 2004. Time-dependent Hurst exponent in financial time series. Physica A, 344, 267-271

Caporin, M., Corazzini, L., Costola, M., 2014. Measuring the Behavioral Component of Financial Fluctuations: An Analysis Based on the S\&P 500, CREATES Research Paper 2014-33. Aarhus University, Denmark

Cochrane, J.H., 2017. Macro-Finance. Review of Finance, European Finance Association, 21(3), 945-985.

Dominitz, J., Manski, C.F., 2005. Measuring And Interpreting Expectations Of Equity Returns. NBER, WP 11313, Cambridge, MA.

Fama, E.F., 1965. The Behavior of Stock-Market Prices. The Journal of Business, 38 (1), 34105.

Fama, E.F., 1970. Efficient Capital Markets: A Review of Theory and Empirical Work, The Journal of Finance, 25 (2), 383-417.

Fama, E.F., 1998. Market efficiency, long-term returns, and behavioral finance, Journal of 
Financial Economics 49, 283-306

Fox, D.R., et.al, 2014. Concepts and Methods of the U.S. National Income and Product Accounts. BEA US DoC, 1-373.

Friedman, B.M., Laibson, D.I., 1989. Economic Implications Of Extraordinary Movements

In Stock Prices. Brookings Papers on Economic Activity, 2, 137-189

Gabaix, X., Gopikrishnan, P., Plerou, V., Stanley, H,E., 2003. A theory of power-law distributions in financial market fluctuations, Nature, 423, 267-270, doi:10.1038/nature01624.

Goldstein, J., 2011. Attractors and Nonlinear Dynamical Systems. Plexus Institute, Washington, DC.

Gontis, V., Havlin, S., Kononovicius, A., Podobnik, B., Stanley, H.E., 2016. Stochastic model of financial markets reproducing scaling and memory in volatility return intervals. Physica A, 462, 1091-1102

Grandmont, J.M., 1992. Expectations Driven Nonlinear Business Cycles, Cowles Foundation Discussion Paper 1022.

Greenwald, D.L., Lettau, M., Ludvigson, S., 2014. Origins of Stock Market Fluctuations. NBER, WP 19818, Cambridge, MA

Greenwood, R., Shleifer, A., 2014. Expectations of Returns and Expected Returns. The Review of Financial Studies, 27 (3), 714-746, https://doi.org/10.1093/rfs/hht082 Guckenheimer, J., Holmes, P., 1990. Nonlinear Oscillations: Dynamical Systems, and Bifurcations of Vector Fields. Berlin, Springer.

Hansen, L.P., Sargent, T.J., 1979. Formulating and Estimating Dynamic Rational Expectations Models. NBER, WP 127

Hansen, L. P., 2013. Uncertainty outside and inside economic models. Nobel lecture. Heaton, J., Lucas, D., 2000. Stock Prices and Fundamentals, in Ed. Bernanke, B.S., Rotemberg, J.J. NBER Macroeconomics Annual 1999, Volume 14, 213 - 264 Henderson, J.V., Squires, T., Storeygard, A., Weil, D., 2016. The Global Spatial Distribution of Economic Activity: Nature, History and the Role of Trade. Spatial Economics Research Center, Discussion Paper 198.

Hördahl, P., Packer,F., 2007. Understanding Asset Prices: an Overview, BIS Papers 34. Horcher, K.A., 2005. Essentials of Financial Risk Management. J.Wiley \& Sons, NJ Hotelling, H., 1929. Stability in Competition. The Economic Journal, 39(153), 41-57 Janžek, T., Ziherl, P., 2013. Overview of models and methods for measuring economic agent's expectations. BIS, IFC Bulletin 36, 172-179. https://www.bis.org/ifc/publ/ifcb36.htm 
Kaihatsu, S., Kurozumi, T., 2010. Sources of Business Fluctuations: Financial or Technology Shocks? Bank of Japan Working Paper Series, No.10-E-12.

Keim, D.B., Stambaugh, R.F., 1986. Predicting Returns In The Stock And Bond Markets, Journal of Financial Economics, 17, 357-390

Kemp, J., 1997. New Methods and Understanding in Economic Dynamics? An Introductory Guide to Chaos and Economics. Economic Issues, 2(1), 1-26

Klaauw, W., de Bruin, W.B., Topa, G., Potter, S., Bryan, M., 2008. Rethinking the Measurement of Household Inflation Expectations: Preliminary Findings. FRB NY,SR 359. Kydland, F., Prescott, E.C., 1980. A Competitive Theory of Fluctuations and the Feasibility and Desirability of Stabilization Policy, 169 - 198 in Ed. Fisher, S., Rational Expectations and Economic Policy, NBER, Univ. Chicago Press

Landau, L.D., Lifshitz, E.M., 1987. Fluid Mechanics. NY: Pergamon Press Ltd Lof, M., 2014. Essays on Expectations and the Econometrics of Asset Pricing, MPRA WP 59064, http://mpra.ub.uni-muenchen.de/59064/

Lorenz, E.N., 1963. Deterministic Nonperiodic Flow. Jour.Atmospheric Sci., 20, 130-141. Loskutov, A., 2010. Fascination of chaos. Uspekhi Fizicheskikh Nauk 180 (12) 1305 -1329 Lucas, R.E., 1972. Expectations and the Neutrality of Money. Jour. of Economic Theory 4, 103-124

Lucas, R.E., 1978. Asset Prices in an Exchange Economy. Econometrica, 46(6), 1429-1445

McNeil, A,J., Frey, R., Embrechts, P., 2005. Quantitative Risk Management. Concepts, Techniques and Tools. Princeton Univ. Press, Princeton.

Mandelbrot, B.B., 1963. The variation of certain speculative prices, J. of Bussiness (Chicago) $36,394-419$.

Mandelbrot, B.B., 1977. Fractals: Form, Chance and Dimension. San-Francisco, Freeman Mandelbrot, B., Fisher, A., Calvet, L., 1997. A Multifractal Model of Asset Returns. Cowles Foundation Discussion Paper \#1164

Manski, C., 2004. Measuring Expectations. Econometrica, 72, 1329-1376.

Manski, C.F., 2017. Survey Measurement Of Probabilistic Macroeconomic Expectations: Progress and Promise. NBER Macro Annual Conference, 1-76.

Muth, J.F., 1961. Rational Expectations and the Theory of Price Movements. Econometrica, 29 (3), 315-335

Neimark, Y. I., Landa, P.S., 1992. Stochastic and Chaotic Oscillations. Dordrecht, Kluver Acad. Publ.

Nicolis, G., Prigogin, I., 1989. Exploring Complexity, an Introduction. W.H. Freeman, NY 
Olkhov, V. (2016a) On Economic space Notion, International Review of Financial Analysis, $47,372-381$

Olkhov, V. (2016b) Finance, Risk and Economic space, ACRN Oxford J. of Finance and Risk Perspectives, Special Issue of Finance Risk and Accounting Perspectives, 5: 209-221

Olkhov, V. (2017a) Quantitative Wave Model of Macro-Finance. International Review of Financial Analysis, 50, 143-150

Olkhov, V. (2017b) Quantitative Description of Financial Transactions and Risks" ACRN Oxford Journal of Finance and Risk Perspectives 6 (2): 41-54.

Olkhov, V. (2017c) Credit-Loans Non-Local Transactions and Surface-Like Waves. https://ssrn.com/abstract=2971417

Olkhov, V. (2017d) Business Cycles: Derivation of Equations for Aggregate Fluctuations, Mean Risks and Mean Square Risks. https://ssrn.com/abstract=3030074

Olkhov, V. (2018). Economic Transactions Govern Business Cycles. MPRA, WP 88461 https://mpra.ub.uni-muenchen.de/88531/

Ott, E., 2002. Chaos in Dynamical Systems. Cambridge Univ. Press.

Pearce, D.K., 1983. Stock Prices and the Economy. FRB of Kansas City, Economic Review, 68 (9), 7-22

Pitman, T., Moss, J., 2016. The Ratings Process. Fitch Ratings, Business Relationship Management, NY Poon, S.H., Granger, C.W.J., 2003. Forecasting Volatility in Financial Markets: A Review Journal of Economic Literature, 41, 478-539

Plerou, V., Gopikrishnan, P., Amaral, L.A.N., Meyer, M., Stanley, H.E., 1999. Scaling of the distribution of price fluctuations of individual companies. Phys. Rev. E, 60 (6), 6519-6529 Plerou, V., Gabaix, X., Gopikrishnan, P., Amaral, L.A.N., Meyer, M., Stanley, H.E., 2001. Price fluctuations, market activity and trading volume. Quantitative Finance, 1, 262-269 Sagdeev, R.Z., Usikov, D.A., Zaslavsky, G.M., 1988. Nonlinear Physics: from the Pendulum to Turbulence and Chaos. Harwood Acad. Publ

Sargent, T.J., Wallace, N., 1976. Rational Expectations And The Theory Of Economic Policy, Journal of Monetary Economics 2 (1976) 169-183

Segnon, M., Lux, T., 2013. Multifractal Models in Finance: Their Origin, Properties, and Applications, WP 1860, Kiel Institute for the World Economy, Kiel, Germany Shiryaev, A.N., 1999. Essentials Of Stochastic Finance. Facts, Models, Theory. World Scientific Publishing Co. Pte. Ltd. London

Skoglund, J., Chen, W., 2015. Financial Risk Management: Applications in Market, Credit, Asset and Liability Management, and Firmwide Risk. John Wiley\&Sons Inc. 
S\&P, 2014. Guide To Credit Rating Essentials. What are credit ratings and how do they work? McGraw Hill Financial

Staff of the U.S. SEC, 2012. Credit Rating Standardization Study. Report to Congress.

Tanaka, M., Bloom, N., David, J.M., Koga, M., 2018. Firm Performance And Macro Forecast Accuracy. NBER WP 24776, Cambridge, MA

Schuster, H.G., Just, W., 2005. Deterministic Chaos. An Introduction. Fourth, Revised and Enlarged Edition. Wiley-VCH Verlag GmbH \& Co. KGaA, Weinheim

Stanley, H.E., Pleroua, V., Gabaixb, X., 2008. A statistical physics view of financial

fluctuations: Evidence for scaling and universality. Physica A, 387, 3967-3981

Starr, R.M., 2011. General Equilibrium Theory, An Introduction, Cam. Univ. NY

Strauss, W.A., 2008. Partial Differential Equations. An Introduction. John Wiley\&Sons, Inc., NJ, US. p.179.

Takens, F., 1981. Dynamical systems and turbulence. Berlin, Springer

Thaler, R.H., 2018. From Cashews to Nudges: The Evolution of Behavioral Economics. American Economic Review, 108(6): 1265-1287

Thompson, J.M.T., Stewart, H.B., 1986. Nonlinear Dynamics and Chaos. Chichester, Wiley Wilier, A.H., 1901. The Economic Theory Of Risk And Insurance. Introduction by Glenn Meyers. Reprinted 1951. Richard D. Irwin, Inc., Homewood, Illinois 